\title{
On the Convergence of Implicit Picard Iterative Sequences for Strongly Pseudocontractive Mappings in Banach Spaces
}

\author{
Shin Min Kang, ${ }^{1}$ Arif Rafiq, ${ }^{2}$ and Sun Young $\mathrm{Cho}^{3}$ \\ ${ }^{1}$ Department of Mathematics and RINS, Gyeongsang National University, Jinju 660-701, Republic of Korea \\ ${ }^{2}$ School of CS and Mathematics, Hajvery University, 43-52 Industrial Area, Gulberg-III, Lahore 54660, Pakistan \\ ${ }^{3}$ Department of Mathematics, Gyeongsang National University, Jinju 660-701, Republic of Korea
}

Correspondence should be addressed to Sun Young Cho; ooly61@yahoo.co.kr

Received 14 December 2012; Accepted 26 March 2013

Academic Editor: Luigi Muglia

Copyright (C) 2013 Shin Min Kang et al. This is an open access article distributed under the Creative Commons Attribution License, which permits unrestricted use, distribution, and reproduction in any medium, provided the original work is properly cited.

We study the convergence of implicit Picard iterative sequences for strongly accretive and strongly pseudocontractive mappings. We have also improved the results of Ćirić et al. (2009).

\section{Introduction and Preliminaries}

Let $E$ be a real Banach space with dual $E^{*}$. The symbol $D(T)$ stands for the domain of $T$.

Let $T: D(T) \rightarrow E$ be a mapping.

Definition 1. The mapping $T$ is said to be Lipschitzian if there exists a constant $L>0$ such that

$$
\|T x-T y\| \leq L\|x-y\|
$$

for all $x, y \in D(T)$.

Definition 2. The mapping $T$ is called strongly pseudocontractive if there exists $t>1$ such that

$$
\|x-y\| \leq\|(1+r)(x-y)-r t(T x-T y)\|
$$

for all $x, y \in D(T)$ and $r>0$. If $t=1$ in inequality (2), then $T$ is called pseudocontractive.

We will denote by $J$ the normalized duality mapping from $E$ to $2^{E^{*}}$ defined by

$$
J(x)=\left\{f^{*} \in E^{*}:\left\langle x, f^{*}\right\rangle=\|x\|^{2}=\left\|f^{*}\right\|^{2}\right\},
$$

where $\langle\cdot, \cdot\rangle$ denotes the generalized duality pairing. It follows from inequality (2) that $T$ is strongly pseudocontractive if and only if there exists $j(x-y) \in J(x-y)$ such that

$$
\langle(I-T) x-(I-T) y, j(x-y)\rangle \geq k\|x-y\|^{2}
$$

for all $x, y \in D(T)$, where $k=(t-1) / t \in(0,1)$. Consequently, from inequality (4) it follows easily that $T$ is strongly pseudocontractive if and only if

$$
\|x-y\| \leq\|x-y+s[(I-T-k I) x-(I-T-k I) y]\|
$$

for all $x, y \in D(T)$ and $s>0$.

Closely related to the class of pseudocontractive maps is the class of accretive operators.

Let $A: D(A) \rightarrow E$ be an operator.

Definition 3. The operator $A$ is called accretive if

$$
\|x-y\| \leq\|x-y+s(A x-A y)\|
$$

for all $x, y \in D(A)$ and $s>0$.

Also, as a consequence of Kato [1], this accretive condition can be expressed in terms of the duality mapping as follows.

For each $x, y \in D(A)$, there exists $j(x-y) \in J(x-y)$ such that

$$
\langle A x-A y, j(x-y)\rangle \geq 0 .
$$


Consequently, inequality (2) with $t=1$ yields that $A$ is accretive if and only if $T:=(I-A)$ is pseudocontractive. Furthermore, from setting $A:=(I-T)$, it follows from inequality (5) that $T$ is strongly pseudocontractive if and only if $(A-k I)$ is accretive, and, using (7), this implies that $T=$ $(I-A)$ is strongly pseudocontractive if and only if there exists $k \in(0,1)$ such that

$$
\langle A x-A y, j(x-y)\rangle \geq k\|x-y\|^{2}
$$

for all $x, y \in D(A)$. The operator $A$ satisfying inequality (8) is called strongly accretive. It is then clear that $A$ is strongly accretive if and only if $T=(I-A)$ is strongly pseudocontractive. Thus, the mapping theory for strongly accretive operators is closely related to the fixed point theory of strongly pseudocontractive mappings. We will exploit this connection in the sequel.

The notion of accretive operators was introduced independently in 1967 by Kato [1] and Browder [2]. An early fundamental result in the theory of accretive operators, due to Browder, states that the initial value problem

$$
\frac{d u}{d t}+A u=0, \quad u(0)=u_{0}
$$

is solvable if $A$ is locally Lipschitzian and accretive on $E$. If $u$ is independent of $t$, then $A u=0$ and the solution of this equation corresponds to the equilibrium points of the system (9). Consequently, considerable research efforts have been devoted, especially within the past 15 years or so, to developing constructive techniques for the determination of the kernels of accretive operators in Banach spaces (see, e.g., [3-19]. Two well-known iterative schemes, the Mann iterative method (see, e.g., [20]) and the Ishikawa iterative scheme (see, e.g., [21]), have successfully been employed.

The Mann and Ishikawa iterative schemes are global and their rate of convergence is generally of the order $O\left(n^{-1 / 2}\right)$. It is clear that if, for an operator $U$, the classical iterative sequence of the form, $x_{n+1}=U x_{n}, x_{0} \in D(U)$ (the socalled Picard iterative sequence) converges, then it is certainly superior and preferred to either the Mann or the Ishikawa sequence since it requires less computations and, moreover, its rate of convergence is always at least as fast as that of a geometric progression.

In $[22,23]$, Chidume proved the following results.

Theorem 4. Let $E$ be an arbitrary real Banach space and $A$ : $E \rightarrow E$ Lipschitz (with constant $L>0$ ) and strongly accretive with a strong accretive constant $k \in(0,1)$. Let $x^{*}$ denote a solution of the equation $A x=0$. Set $\epsilon:=(1 / 2)(k /(1+L(3+$ $L-k))$ ) and define $A_{\epsilon}: E \rightarrow E$ by $A_{\epsilon} x:=x-\epsilon A x$ for each $x \in E$.

For arbitrary $x_{0} \in E$, define the sequence $\left\{x_{n}\right\}_{n=0}^{\infty}$ in $E$ by

$$
x_{n+1}=A_{\epsilon} x_{n}, \quad n \geq 0 .
$$

Then $\left\{x_{n}\right\}_{n=0}^{\infty}$ converges strongly to $x^{*}$ with

$$
\left\|x_{n+1}-x^{*}\right\| \leq \delta^{n}\left\|x_{0}-x^{*}\right\|
$$

where $\delta=(1-(1 / 2) k \epsilon) \in(0,1)$. Moreover, $x^{*}$ is unique.
Corollary 5. Let $E$ be an arbitrary real Banach space and $K$ a nonempty convex subset of $E$. Let $T: K \rightarrow K$ be Lipschitz (with constant $L>0$ ) and strongly pseudocontractive (i.e., $T$ satisfies inequality (5) for all $x, y \in K)$. Assume that $T$ has a fixed point $x^{*} \in K$. Set $\epsilon_{0}:=(1 / 2)(k /(1+L(3+L-k)))$ and define $T_{\epsilon_{0}}: K \rightarrow K$ by $T_{\epsilon_{0}} x=\left(1-\epsilon_{0}\right) x+\epsilon_{0} T x$ for each $x \in K$. For arbitrary $x_{0} \in K$, define the sequence $\left\{x_{n}\right\}_{n=0}^{\infty}$ in $K$ by

$$
x_{n+1}=T_{\epsilon_{0}} x_{n}, \quad n \geq 0 .
$$

Then $\left\{x_{n}\right\}_{n=0}^{\infty}$ converges strongly to $x^{*}$ with

$$
\left\|x_{n+1}-x^{*}\right\| \leq \delta^{n}\left\|x_{0}-x^{*}\right\|
$$

where $\delta=\left(1-(1 / 2) k \epsilon_{0}\right) \in(0,1)$. Moreover, $x^{*}$ is unique.

Recently, Ćirić et al. [24] improved the results of Chidume $[22,23]$, Liu [14], and Sastry and Babu [18] as in the following results.

Theorem 6. Let $E$ be an arbitrary real Banach space and $A$ : $E \rightarrow E$ a Lipschitz (with constant $L>0$ ) and strongly accretive with a strong accretive constant $k \in(0,1)$. Let $x^{*}$ denote a solution of the equation $A x=0$. Set $\varepsilon:=(k-\eta) / L(2+L)$, $\eta \in(0, k)$ and define $A_{\varepsilon}: E \rightarrow E$ by $A_{\varepsilon} x:=x-\varepsilon A x$ for each $x \in E$. For arbitrary $x_{0} \in E$, define the sequence $\left\{x_{n}\right\}_{n=0}^{\infty}$ in $E$ by

$$
x_{n+1}=A_{\varepsilon} x_{n}, \quad n \geq 0 .
$$

Then $\left\{x_{n}\right\}_{n=0}^{\infty}$ converges strongly to $x^{*}$ with

$$
\left\|x_{n+1}-x^{*}\right\| \leq \theta^{n}\left\|x_{0}-x^{*}\right\|
$$

where $\theta=(1-((k-\eta) /(k(k-\eta)+L(2+L))) \eta) \in(0,1)$. Thus the choice $\eta=k / 2$ yields $\theta=1-k^{2} / 2[k+2 L(2+L)]$. Moreover, $x^{*}$ is unique.

Corollary 7. Let $E$ be an arbitrary real Banach space and $K$ a nonempty convex subset of $E$. Let $T: K \rightarrow K$ be Lipschitz (with constant $L>0$ ) and strongly pseudocontractive (i.e., $T$ satisfies inequality (5) for all $x, y \in K$ ). Assume that $T$ has a fixed point $x^{*} \in K$. Set $\varepsilon_{0}:=(k-\eta) / L(2+L), \eta \in(0, k)$ and define $T_{\varepsilon_{0}}: K \rightarrow K$ by $T_{\varepsilon_{0}} x=\left(1-\varepsilon_{0}\right) x+\varepsilon_{0} T x$ for each $x \in K$. For arbitrary $x_{0} \in K$, define the sequence $\left\{x_{n}\right\}_{n=0}^{\infty}$ in $K$ by

$$
x_{n+1}=T_{\varepsilon_{0}} x_{n}, \quad n \geq 0 .
$$

Then $\left\{x_{n}\right\}_{n=0}^{\infty}$ converges strongly to $x^{*}$ with

$$
\left\|x_{n+1}-x^{*}\right\| \leq \theta^{n}\left\|x_{0}-x^{*}\right\|
$$

where $\theta=(1-((k-\eta) /(k(k-\eta)+L(2+L))) \eta) \in(0,1)$. Moreover, $x^{*}$ is unique.

In this paper, we study the convergence of implicit Picard iterative sequences for strongly accretive and strongly pseudocontractive mappings. We have also improved the results of Ćirić et al. [24]. 


\section{Main Results}

In the following theorems, $L>1$ will denote the Lipschitz constant of the operator $A$ and $k$ will denote the strong accretive constant of the operator $A$ as in inequality (8). Furthermore, $\epsilon>0$ is defined by

$$
\epsilon:=\frac{k-\eta}{L+(1+L)(k-\eta)}, \quad \eta \in(0, k) .
$$

With these notations, we prove the following theorem.

Theorem 8. Let $E$ be an arbitrary real Banach space and $A$ : $E \rightarrow E$ Lipschitz and strongly accretive with a strong accretive constant $k \in(0,1)$. Let $x^{*}$ denote a solution of the equation $A x=0$. Define $A_{\epsilon}: E \rightarrow E$ by $A_{\epsilon} x_{n}=(1-\epsilon) x_{n-1}+\epsilon x_{n}-\epsilon A x_{n}$ for each $x_{n} \in E$. For arbitrary $x_{0} \in E$, define the sequence $\left\{x_{n}\right\}_{n=0}^{\infty}$ in $E$ by

$$
x_{n}=A_{\epsilon} x_{n}, \quad n \geq 1 .
$$

Then $\left\{x_{n}\right\}_{n=0}^{\infty}$ converges strongly to $x^{*}$ with

$$
\left\|x_{n+1}-x^{*}\right\| \leq \rho^{n}\left\|x_{0}-x^{*}\right\|
$$

where $\rho=(1-((k-\eta) /(L+((k-\eta)(1+L+k))) \eta) \in(0,1)$. Thus the choice $\eta=k / 2$ yields $\rho=1-k^{2} / 2[2 L+k(1+L+k)]$. Moreover, $x^{*}$ is unique.

Proof. Existence of $x^{*}$ follows from [5, Theorem 13.1]. Define $T=(I-A)$ where $I$ denotes the identity mapping on $E$. Observe that $A x^{*}=0$ if and only if $x^{*}$ is a fixed point of $T$. Moreover, $T$ is strongly pseudocontractive since $A$ is strongly accretive, and so $T$ also satisfies inequality (5) for all $x, y \in E$ and $s>0$. Furthermore, the recursion formula $x_{n}=A_{\epsilon} x_{n}$ becomes

$$
x_{n}=(1-\epsilon) x_{n-1}+\epsilon T x_{n}, \quad n \geq 1 \text {. }
$$

Observe that

$$
x^{*}=(1+\epsilon) x^{*}+\epsilon(I-T-k I) x^{*}-(1-k) \epsilon x^{*},
$$

and from the recursion formula (21)

$$
\begin{aligned}
x_{n-1}= & (1+\epsilon) x_{n}+\epsilon(I-T-k I) x_{n} \\
& -(1-k) \epsilon x_{n}+\epsilon^{2}\left(x_{n-1}-T x_{n}\right),
\end{aligned}
$$

which implies that

$$
\begin{aligned}
x_{n-1}-x^{*}= & (1+\epsilon)\left(x_{n}-x^{*}\right) \\
& +\epsilon\left[(I-T-k I) x_{n}-(I-T-k I) x^{*}\right] \\
& -(1-k) \epsilon\left(x_{n}-x^{*}\right)+\epsilon^{2}\left(x_{n-1}-T x_{n}\right) .
\end{aligned}
$$

This implies using inequality (5) with $s=\epsilon /(1+\epsilon)$ and $y=x^{*}$ that

$$
\begin{aligned}
& \left\|x_{n-1}-x^{*}\right\| \\
& \geq(1+\epsilon)\left(\|\left(x_{n}-x^{*}\right)+\frac{\epsilon}{1+\epsilon}\right. \\
& \left.\quad \times\left[(I-T-k I) x_{n}-(I-T-k I) x^{*}\right] \|\right) \\
& \quad-(1-k) \epsilon\left\|x_{n}-x^{*}\right\|-\epsilon^{2}\left\|x_{n-1}-T x_{n}\right\| \\
& \geq \quad(1+\epsilon)\left\|x_{n}-x^{*}\right\|-(1-k) \epsilon\left\|x_{n}-x^{*}\right\| \\
& \quad-\epsilon^{2}\left\|x_{n-1}-T x_{n}\right\| \\
& =(1+k \epsilon)\left\|x_{n}-x^{*}\right\|-\epsilon^{2}\left\|x_{n-1}-T x_{n}\right\| .
\end{aligned}
$$

Observe that

$$
\begin{aligned}
\left\|x_{n-1}-T x_{n}\right\| & \leq\left\|x_{n-1}-T x_{n-1}\right\|+\left\|T x_{n-1}-T x_{n}\right\| \\
& \leq\left\|A x_{n-1}\right\|+\left\|x_{n-1}-x_{n}\right\|+\left\|A x_{n-1}-A x_{n}\right\| \\
& \leq L\left\|x_{n-1}-x^{*}\right\|+(1+L)\left\|x_{n-1}-x_{n}\right\| \\
& =L\left\|x_{n-1}-x^{*}\right\|+(1+L) \epsilon\left\|x_{n-1}-T x_{n}\right\|,
\end{aligned}
$$

and so

$$
\left\|x_{n-1}-T x_{n}\right\| \leq \frac{L}{1-(1+L) \epsilon}\left\|x_{n-1}-x^{*}\right\|,
$$

so that from (25) we obtain

$$
\begin{aligned}
\left\|x_{n-1}-x^{*}\right\| \geq & (1+k \epsilon)\left\|x_{n}-x^{*}\right\| \\
& -\frac{L \epsilon^{2}}{1-(1+L) \epsilon}\left\|x_{n-1}-x^{*}\right\| .
\end{aligned}
$$

Therefore

$$
\left\|x_{n}-x^{*}\right\| \leq \frac{1+L \epsilon^{2} /(1-(1+L) \epsilon)}{1+k \epsilon}\left\|x_{n-1}-x^{*}\right\|,
$$

where

$$
\begin{aligned}
\rho & =\frac{1+L \epsilon^{2} /(1-(1+L) \epsilon)}{1+k \epsilon} \\
& =1-\frac{\epsilon}{1+k \epsilon}\left(k-\frac{L \epsilon}{1-(1+L) \epsilon}\right)=1-\frac{\epsilon}{1+k \epsilon} \eta \\
& =1-\frac{k-\eta}{L+(k-\eta)(1+L+k)} \eta .
\end{aligned}
$$

From (29) and (30), we get

$$
\begin{aligned}
\left\|x_{n}-x^{*}\right\| & \leq \rho\left\|x_{n-1}-x^{*}\right\| \\
& \leq \cdots \\
& \leq \rho^{n}\left\|x_{0}-x^{*}\right\| \\
& \longrightarrow 0
\end{aligned}
$$


as $n \rightarrow \infty$. Hence $x_{n} \rightarrow x^{*}$ as $n \rightarrow \infty$. Uniqueness follows from the strong accretivity property of $A$.

The following is an immediate corollary of Theorem 8 .

Corollary 9. Let $E$ be an arbitrary real Banach space and $K$ a nonempty convex subset of E. Let $T: K \rightarrow K$ be Lipschitz (with constant $L>1$ ) and strongly pseudocontractive (i.e., $T$ satisfies inequality (5) for all $x, y \in K)$. Assume that $T$ has a fixed point $x^{*} \in K$. Set $\epsilon_{0}:=(k-\eta) /(L+(1+L)(k-\eta))$, $\eta \in(0, k)$ and define $A_{\epsilon_{0}}: K \rightarrow K$ by $A_{\epsilon_{0}} x_{n}=\left(1-\epsilon_{0}\right) x_{n-1}+$ $\epsilon_{0} x_{n}-\epsilon_{0} A x_{n}$ for each $x_{n} \in K$. For arbitrary $x_{0} \in K$, define the sequence $\left\{x_{n}\right\}_{n=0}^{\infty}$ in $K$ by

$$
x_{n}=A_{\epsilon_{0}} x_{n}, \quad n \geq 1 .
$$

Then $\left\{x_{n}\right\}_{n=0}^{\infty}$ converges strongly to $x^{*}$ with

$$
\left\|x_{n+1}-x^{*}\right\| \leq \rho_{0}^{n}\left\|x_{0}-x^{*}\right\|
$$

where $\rho_{0}=(1-((k-\eta) /(L+(k-\eta)(1+L+k))) \eta) \in(0,1)$. Thus the choice $\eta=k / 2$ yields $\rho_{0}=1-k^{2} / 2[2 L+k(1+L+k)]$. Moreover, $x^{*}$ is unique.

Proof. Observe that $x^{*}$ is a fixed point of $T$ if and only if it is a fixed point of $T_{\epsilon_{0}}$. Furthermore, the recursion formula (32) is simplified to the formula

$$
x_{n}=\left(1-\epsilon_{0}\right) x_{n-1}+\epsilon_{0} T x_{n},
$$

which is similar to (21). Following the method of computations as in the proof of the Theorem 8 , we obtain

$$
\begin{aligned}
\left\|x_{n}-x^{*}\right\| & \leq \frac{1+L \epsilon_{0}^{2} /\left(1-(1+L) \epsilon_{0}\right)}{1+k \epsilon_{0}}\left\|x_{n-1}-x^{*}\right\| \\
& =\left(1-\frac{k-\eta}{L+(k-\eta)(1+L+k)} \eta\right)\left\|x_{n-1}-x^{*}\right\| .
\end{aligned}
$$

Set $\rho_{0}=1-((k-\eta) /(L+(k-\eta)(1+L+k))) \eta$. Then from (35) we obtain

$$
\begin{aligned}
\left\|x_{n}-x^{*}\right\| & \leq \rho_{0}\left\|x_{n-1}-x^{*}\right\| \\
& \leq \cdots \\
& \leq \rho_{0}^{n}\left\|x_{0}-x^{*}\right\| \\
& \longrightarrow 0
\end{aligned}
$$

as $n \rightarrow \infty$. This completes the proof.

Remark 10. Since $L>1$ and $k<L$, we have

$$
L>k-\eta
$$

So we can easily obtain

$$
\frac{1}{L+(k-\eta)(1+L+k)}>\frac{1}{k(k-\eta)+L(2+L)} \text {. }
$$

Now

$$
\begin{aligned}
\rho & =1-\frac{(k-\eta)}{L+(k-\eta)(1+L+k)} \eta \\
& <1-\frac{(k-\eta)}{k(k-\eta)+L(2+L)} \eta \\
& =\theta .
\end{aligned}
$$

Thus the relation between Ćirić et al. [24] and our parameter of convergence, that is, between $\theta$ and $\rho$, respectively, is the following:

$$
\rho<\theta
$$

Our convergence parameter $\rho$ shows the overall improvement for $\theta$, and consequently the results of Ćirić et al. [24] are improved.

\section{Acknowledgment}

The authors would like to thank the referees for useful comments and suggestions.

\section{References}

[1] T. Kato, "Nonlinear semigroups and evolution equations," Journal of the Mathematical Society of Japan, vol. 19, pp. 508$520,1967$.

[2] F. E. Browder, "Nonlinear mappings of nonexpansive and accretive type in Banach spaces," Bulletin of the American Mathematical Society, vol. 73, pp. 875-882, 1967.

[3] C. E. Chidume, "Iterative approximation of fixed points of Lipschitzian strictly pseudocontractive mappings," Proceedings of the American Mathematical Society, vol. 99, no. 2, pp. 283$288,1987$.

[4] C. E. Chidume, "An iterative process for nonlinear Lipschitzian strongly accretive mappings in $L_{p}$ spaces," Journal of Mathematical Analysis and Applications, vol. 151, no. 2, pp. 453-461, 1990.

[5] K. Deimling, Nonlinear Functional Analysis, Springer, Berlin, Germany, 1985.

[6] L. Deng, "On Chidume's open questions," Journal of Mathematical Analysis and Applications, vol. 174, no. 2, pp. 441-449, 1993.

[7] L. Deng, "An iterative process for nonlinear Lipschitzian and strongly accretive mappings in uniformly convex and uniformly smooth Banach spaces," Acta Applicandae Mathematicae, vol. 32, no. 2, pp. 183-196, 1993.

[8] L. Deng, "Iteration processes for nonlinear Lipschitzian strongly accretive mappings in $L_{p}$ spaces," Journal of Mathematical Analysis and Applications, vol. 188, no. 1, pp. 128-140, 1994.

[9] L. Deng and X. P. Ding, "Iterative approximation of Lipschitz strictly pseudocontractive mappings in uniformly smooth Banach spaces," Nonlinear Analysis, vol. 24, no. 7, pp. 981-987, 1995.

[10] N. Hussain, L. B. Ćirić, Y. J. Cho, and A. Rafiq, "On Manntype iteration method for a family of hemicontractive mappings in Hilbert spaces," Journal of Inequalities and Applications, vol. 2013, article 41, 11 pages, 2013. 
[11] N. Hussain, A. Rafiq, and L. B. Ćirić, "Stability of the Ishikawa iteration scheme with errors for two strictly hemicontractive operators in Banach spaces," Fixed Point Theory and Applications, vol. 2012, article 160, 14 pages, 2012.

[12] N. Hussain, A. Rafiq, L. B. Ćirić, and S. Al-Mezel, "Almost stability of the Mann type iteration method with error term involving strictly hemicontractive mappings in smooth Banach spaces," Journal of Inequalities and Applications, vol. 2012, article 207, 11 pages, 2012.

[13] N. Hussain, A. Rafiq, B. Damjanović, and R. Lazović, "On rate of convergence of various iterative schemes," Fixed Point Theory and Applications, vol. 2011, article 45, 6 pages, 2011.

[14] L. Liu, "Approximation of fixed points of a strictly pseudocontractive mapping," Proceedings of the American Mathematical Society, vol. 125, no. 5, pp. 1363-1366, 1997.

[15] Q. H. Liu, "The convergence theorems of the sequence of Ishikawa iterates for hemicontractive mappings," Journal of Mathematical Analysis and Applications, vol. 148, no. 1, pp. 5562, 1990.

[16] A. Rafiq, "On Mann iteration in Hilbert spaces," Nonlinear Analysis, vol. 66, no. 10, pp. 2230-2236, 2007.

[17] A. Rafiq, "Implicit fixed point iterations for pseudocontractive mappings," Kodai Mathematical Journal, vol. 32, no. 1, pp. 146158, 2009.

[18] K. P. R. Sastry and G. V. R. Babu, "Approximation of fixed points of strictly pseudocontractive mappings on arbitrary closed, convex sets in a Banach space," Proceedings of the American Mathematical Society, vol. 128, no. 10, pp. 2907-2909, 2000.

[19] X. L. Weng, "Fixed point iteration for local strictly pseudocontractive mapping," Proceedings of the American Mathematical Society, vol. 113, no. 3, pp. 727-731, 1991.

[20] W. R. Mann, "Mean value methods in iteration," Proceedings of the American Mathematical Society, vol. 4, pp. 506-510, 1953.

[21] S. Ishikawa, "Fixed points by a new iteration method," Proceedings of the American Mathematical Society, vol. 44, pp. 147-150, 1974.

[22] C. E. Chidume, "Picard iteration for strongly accretive and strongly pseudocontractive Lipschitz maps," International Centre for Theoretical Physics. In press.

[23] C. E. Chidume, "Iterative algorithms for nonexpansive mappings and some of their generalizations," in Nonlinear Analysis and Applications: to V. Lakshmikantham on His 80th Birthday, vol. 1,2, pp. 383-429, Kluwer Academic, Dordrecht, The Netherlands, 2003.

[24] L. Ćirić, A. Rafiq, and N. Cakić, "On Picard iterations for strongly accretive and strongly pseudo-contractive Lipschitz mappings," Nonlinear Analysis, vol. 70, no. 12, pp. 4332-4337, 2009. 


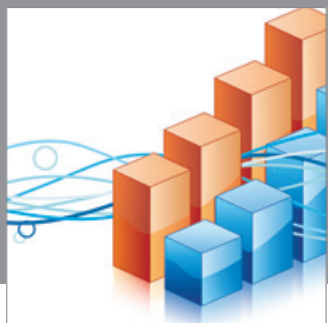

Advances in

Operations Research

mansans

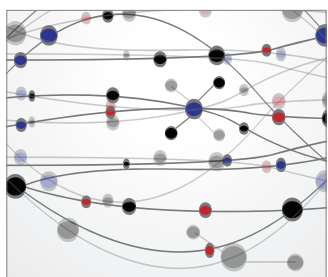

The Scientific World Journal
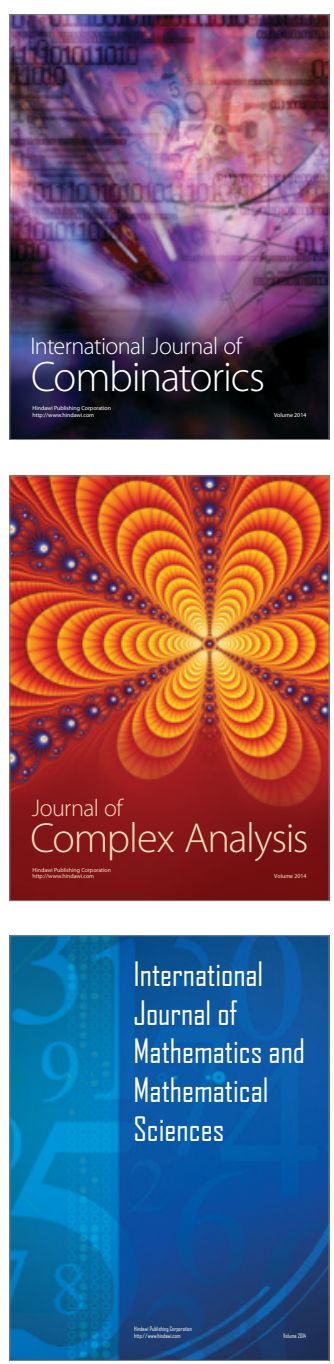
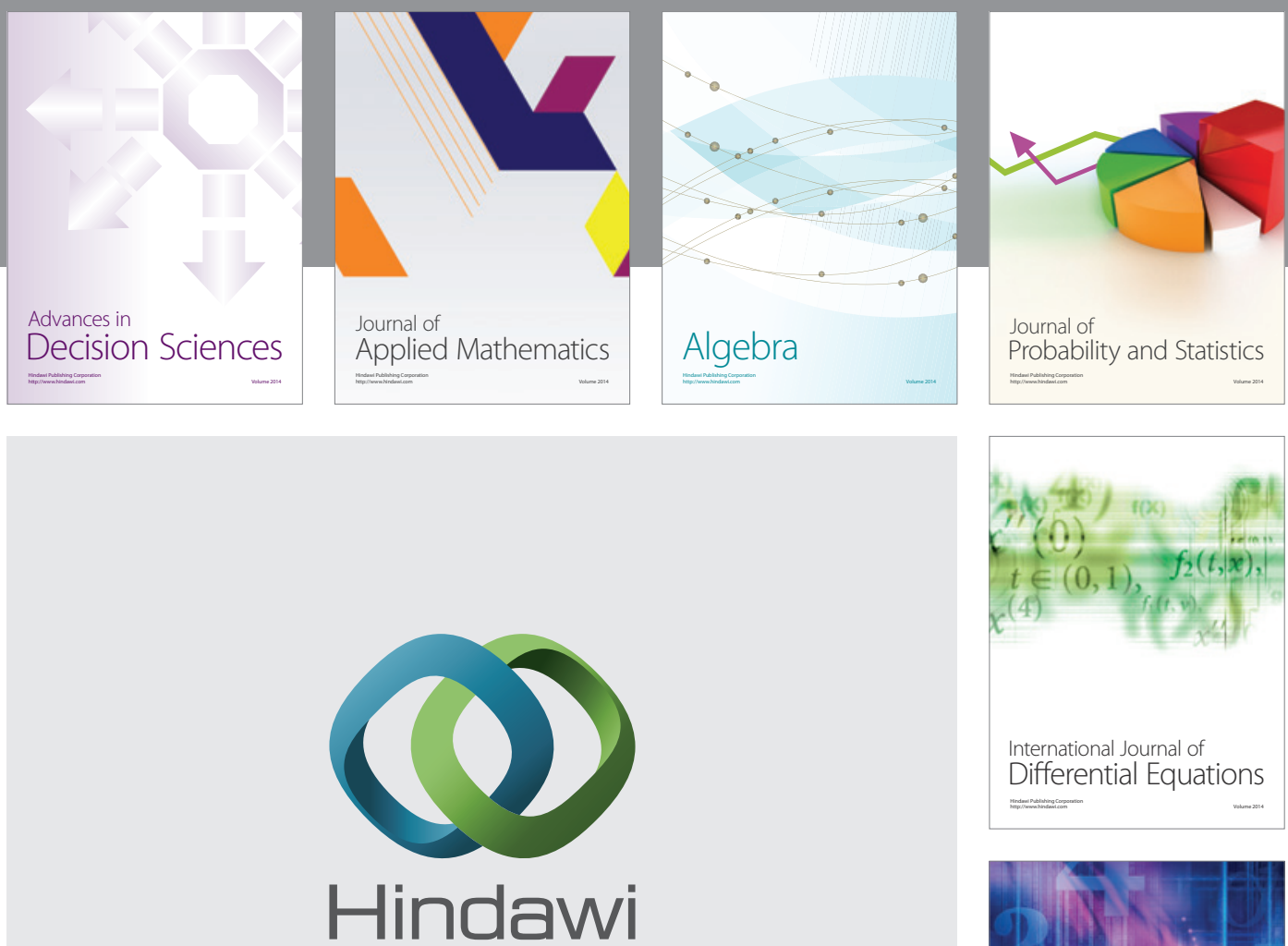

Submit your manuscripts at http://www.hindawi.com


Journal of

Function Spaces

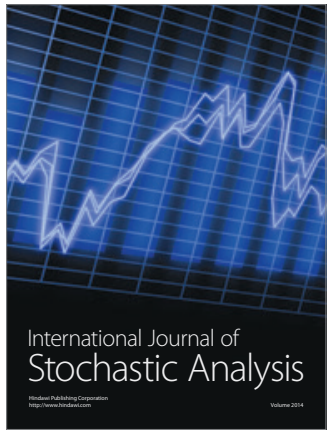


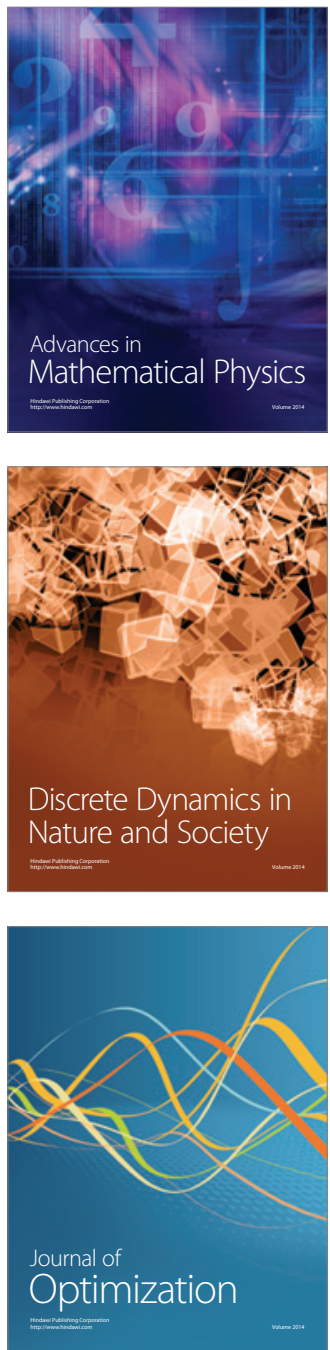